\title{
Editorial
}

\section{Genetic Therapy for Pancreatic Cancer: Is it the Future?}

\author{
Lorenzo Dioscoridi, MD* \\ Departments of Surgery and Translational Medicine, University of Florence, Firenze, Italy \\ ${ }^{*}$ Corresponding author \\ Lorenzo Dioscoridi, MD \\ Departments of Surgery and Translational Medicine, University of Florence, Firenze, Italy; E-mail: dioscoridi.lorenzo@virgilio.it
}

Article information

Received: August 4th 2016 ; Accepted: August 4 $4^{\text {th }}, 2016$; Published: August $5^{\text {th }}, 2016$

Cite this article

Dioscoridi L. Genetic therapy for pancreatic cancer: Is it the future? Pancreas Open J. 20I6; 2(I): el-e2. doi: I0.I7I40/POJ-2-e005

lthough surgery is still the only curative therapy for pancreAatic cancer, oncological therapies have started to be more and more promising.

We firstly have to consider two important points. The first one is that surgery for pancreatic cancer is complex and risky: perioperative mortality is $4-18 \%$ and major complications occur in more than $40 \%$ of cases. ${ }^{1}$ Moreover, only $15-20 \%$ of patients have a resectable tumour at the first diagnosis. ${ }^{2}$ Furthermore, disease's recurrence following operation is very high because of local invasion and micrometastases at the time of surgery. ${ }^{3}$ The second point is that the first chemotherapies for pancreatic cancer (5-fluoro-uracile, and the actually used, gemcitabine) are not specific for pancreatic tumours and have been provided only a little impact on overall survive. $^{4}$

The actually used chemotherapies are gemcitabine, the association of gemcitabine and nab-paclitaxel (that improved overall survival, progression free survival and response rate compared to gemcitabine alone) and the recent FOLFIRINOX (oxaliplatin, irinotecan, leucovorin) that have been shown to be more efficient than gemcitabine. ${ }^{5}$

To obtain more efficient chemotherapies, we have to comprehend the molecular biology of pancreatic cancer.

Recent articles have shown the principal gene mutations in pancreatic tumoural cells. The earlier events involve telomere shortening, KRAS (Kirsten rat sarcoma viral oncogene homolog) mutation and p16 loss; the later ones are mainly P53 loss, SMAD4 (SMAD family member 4) loss and BRCA2 (breast cancer 2) mutations. $^{3}$

Pancreatic cancer also overexpress multiple mitogenetic growth factors and their ligands i.e. epidermal growth factor receptor (EGFR).
Other studies have underlined the importance of the developmental program of epithelial to mesenchymal transition (EMT) especially for metastatisation. This process is regulated by inducing transcription factors as TGF-beta, Wnt/beta-catenin, Notch signaling, Snail transcription factors, zinc-finger-enhancer binding (Zeb) protein family and basic helix loop helix (bHLH) transcription factors. ${ }^{7}$

Recently, the role of cancer stem cells has been found to be responsible for tumour recurrence and metastases. Notch, Hedgehog and Wnt/beta-catenin signaling pathways have a role in maintaining stem cells' line in pancreatic cancer.,

All these new knowledges have extended the scientific research about therapies that can specifically interrupt the tumoural development.

Thus, K-ras has been choosen as an important target trying to block K-ras membrane localization (i.e. Deltarisin) or SOS/ $\mathrm{K}$-ras interaction or K-ras downstream effector targets (i.e. sulfinyl cytidine). ${ }^{10}$

Another considered target is HDAC (histone deacetylase). It is involved in Snail mediated epidermal to mesenchymal process. Many histone deacetylase inhibitors have been tested in preclinical and early clinical studies. ${ }^{11}$

Targeting cancer stem cells is being considered and this is the recent therapeutical concept. ${ }^{12}$ Hedgehog inhibitors are under preclinical and clinical development. Notch inhibitors seem to be more effective than gemcitabine and monoclonal antibodies that has been studied. ${ }^{12}$

In conclusion, pancreatic cancer biology is better understood than 10 years ago. This is the first step to develop a specific genetic therapy. Surgery is still the only curative therapy but it has many important limitations. Therefore, it cannot be considered the 
future definitive therapy. However, there are no effective therapies available because the multifactoral nature of the tumour i.e. we will have to consider the different types of pancreatic cancer on the base of different gene mutations and we may finally find a curative therapy.

\section{REFERENCES}

1. Freelove R, Walling AD. Pancreatic cancer: Diagnosis and management. Am Fam Physician. 2006; 73: 485-492. Web site. http:// www.aafp.org/afp/2006/0201/p485.html. Accessed August 3, 2016

2. Li D, Xie K, Wolff R, Abruzzese JL. Pancreatic cancer. Lancet. 2004; 363: 1049-1057. doi: 10.1016/S0140-6736(04)15841-8

3. Polireddy K, Chen Q. Cancer of the pancreas: Molecular pathways and current advancement in treatment. J Cancer. 2016; 7 : 1497-1514. doi: 10.7150/jca.14922

4. Herman JM, Swartz MJ, Hsu CC, et al. Analysis of fluorouracil-based adjuvant chemotherapy and radiation after pancreaticoduodenectomy for ductal adenocarcinoma of the pancreas: Results of a large, prospectively collected database at the Johns Hopkins Hospital. J Clin Oncol. 2008; 26: 3503-3510. doi: 10.1200/ JCO.2007.15.8469

5. Conroy T, Desseigne F, Ychou M, et al. FOLFIRINOX versus gemcitabine for metastatic pancreatic cancer. N Engl J Med. 2011;
364: 1817-1825. doi: 10.1056/NEJMoa1011923

6. Troiani T, Martinelli E, Capasso A, et al. Targeting EGFR in pancreatic cancer treatment. Curr Drug Targets. 2012; 13: 802-810. doi: 10.2174/138945012800564158

7. Rhim AD, Mirek ET, Aiello NM, et al. EMT and dissemination precede pancreatic tumour formation. Cell. 2012; 148: 349-361. doi: 10.1016/j.cell.2011.11.025

8. Kreso A, Dick JE. Evolution of the cancer stem cell model. Cell Stem Cell. 2014; 14: 275-291. doi: 10.1016/j.stem.2014.02.006

9. Simeone DM. Pancreatic cancer stem cells: Implications for the treatment of pancreatic cancer. Clin Cancer Res. 2008; 14: 56465648. doi: 10.1158/1078-0432.CCR-08-0584

10. Bryant KL, Mancias JD, Kimmelman AC, Der CJ. KRAS: Feeding pancreatic cancer proliferation. Trends Biochem Sci. 2014; 39: 91 100. doi: $10.1016 /$ j.tibs.2013.12.004

11. Fritsche P, Seidler B, Schuler S, et al. HDAC2 mediates therapeutic resistance of pancreatic cancer cells via the $\mathrm{BH}-3$ only protein NOXA. Gut. 2009; 58: 1399-1409. doi: 10.1136/gut.2009.180711

12. Tang SC, Chen YC. Novel therapeutic targets for pancreatic cancer. World J Gastroenterol. 2014; 20: 10825-10844. doi: 10.3748/ wjg.v20.i31.10825 